\title{
Orthopedic and Esthetic Resolution of a Severe Case of Amelogenesis Imperfecta (Type I-Hypoplasic)
}

\author{
${ }^{1}$ Matheus Melo Pithon, ${ }^{2}$ Hazarmavé Barreto Pinto, ${ }^{3}$ Rajiv Saini
}

\begin{abstract}
Amelogenesis imperfecta is a genetically transmitted tooth enamel malformation that may affect both primary and permanent dentition. It is of a hereditary nature and is limited to the tooth enamel structure, without the presence of other apparent systemic compromise. The authors' purpose in the present article is to describe the clinical case of a patient with severe amelogenesis imperfecta treated facial orthopedics and esthetic reconstruction of the teeth. The results achieved on conclusion of treatment were the correction of the patient's maxillary atresia and improvement of dental and facial esthetics, providing the patient with greater social and psychological benefits.
\end{abstract}

Keywords: Amelogenesis imperfecta, Tooth enamel, Esthetics.

How to cite this article: Pithon MM, Pinto HB, Saini R. Orthopedic and Esthetic Resolution of a Severe Case of Amelogenesis Imperfecta (Type I-Hypoplasic). Int J Experiment Dent Sci 2014;3(2):109-114.

\section{Source of support: Nil}

Conflict of interest: None

\section{INTRODUCTION}

Amelogenesis imperfecta is a genetically transmitted tooth enamel malformation that may affect both primary and permanent dentition. This alteration in amelogenesis is of a hereditary nature and is limited to the tooth enamel structure, without the presence of other apparent systemic compromise. Diagnosis is generally made through clinical studies and information obtained from the family itself. ${ }^{1-3}$

Generally speaking, local or systemic disturbances during formation of the enamel organ may produce alterations to its structure. These alterations may be relative to a defect in the quantity (reduction in thickness causing hypoplasia) or in the quality (hypomineralization causing opacities) of the tooth enamel. ${ }^{1-3}$

\footnotetext{
${ }^{1}$ Professor, ${ }^{2}$ Student, ${ }^{3}$ Associate Professor

${ }^{1,2}$ Department of Orthodontics, Southwest Bahia State University-UESB, Bahia, Brazil

${ }^{3}$ Department of Periodontology, Pravara Institute of Medical Sciences, Ahmednagar, Maharashtra, India

Corresponding Author: Matheus Melo Pithon, Professor Department of Orthodontics, Southwest Bahia State University-UESB, Bahia, Brazil, Phone: 557730842020 e-mail: matheuspithon@gmail.com
}

The phase of activity of the ameloblast, duration of the disturbance and its intensity indicate the temporary or permanent inactivity of cells, thus determining the appearance of the most commonly found types. ${ }^{1}$

Therefore, in a simplified manner, amelogenesis imperfecta has been divided into four groups: type I — hypoplasic; type II - hypomaturated; type III - hypocalcified; type IV - hypomaturated-hypoplasic with taurodontism. There is a variation ranging from an aspect of deficient enamel through to episodes of defects in the mineral and protein substance. $^{4-7}$

In spite of many studies having revealed prevalence values of AI with great disparity among them, there is some consensus with respect to an incidence of 1 in every 14,000 individuals ${ }^{4,6}$ and a higher incidence of the hypocalcified type of AI, followed by the types by hypomaturation and hypoplasia. ${ }^{6}$

The main clinical problems in patients affected by the disease are the loss of large extensions of dental tissue, compromised function, and dental sensitivity, however, it is important to point out that the most evident of the disorders caused by this dental disturbance is deficient esthetics, which my lead to severe psychological and social problems for the affected patient. ${ }^{5}$

From this aspect, with the development of dental materials, today it is possible to rehabilitate these cases with less invasive procedures, with predictability and longevity of the results obtained, restoring the patient's self-esteem and wellbeing, factors that justify early intervention in these cases. ${ }^{8}$

Therefore, the authors' proposal in the present study is to relate the orthopedic and esthetic resolution of a case of severe amelogenesis imperfecta, in which there was remarkable esthetic compromise.

\section{CASE REPORT}

The patient RFS, a 12-year-old girl, melanoderma, was brought to the Clinic of the Pediatric Dentistry Course of the Dentistry course at the State University of Southeast Bahia by her mother, with the chief complaint that she experienced 'pain in her teeth when she ingested foods, and had poor dental esthetics'. After anamnesis and clinical exam, accentuated wear of the teeth, with a rough aspect and yellow stains, in addition to dental sensitivity and diastemas were observed, which made the girl reluctant to smile. The patient 
had a good state of health, without any systemic change. Yet the same was referred to a general medical clinic testified that the general health of the same Figure 1. With regard to occlusion, the patient presented malocclusion class I, anteroposterior open bite and posterior bilateral cross bite (cephalometrically the patient had a relationship class II with $\mathrm{SNA}=71^{\circ}, \mathrm{SNB}=66^{\circ}$ eANB $=5^{\circ}$ ). Because of the lack of point contacts migration occurred prior to inferiors teeth following the trend of shifting teeth mesial favoring a class I malocclusion. Routine preventive procedures were performed, and the patient was diagnosed with amelo- genesis imperfecta of the hypoplasic type in the maxillary and mandibular arches.

To prove the clinically seen findings, the patient's documentation was prepared by taking panoramic radiographs, intra- and extrabuccal photographs, performing cephalometry and making study models (Figs 2 and 3).

The treatment plan proposed for the case was the association of restorative and orthopedic procedures.

In the beginning of orthodontic treatment, priority was given to restoring the functionality of the teeth, in which reconstruction of the crowns of posterior teeth $(16,14,24,26)$
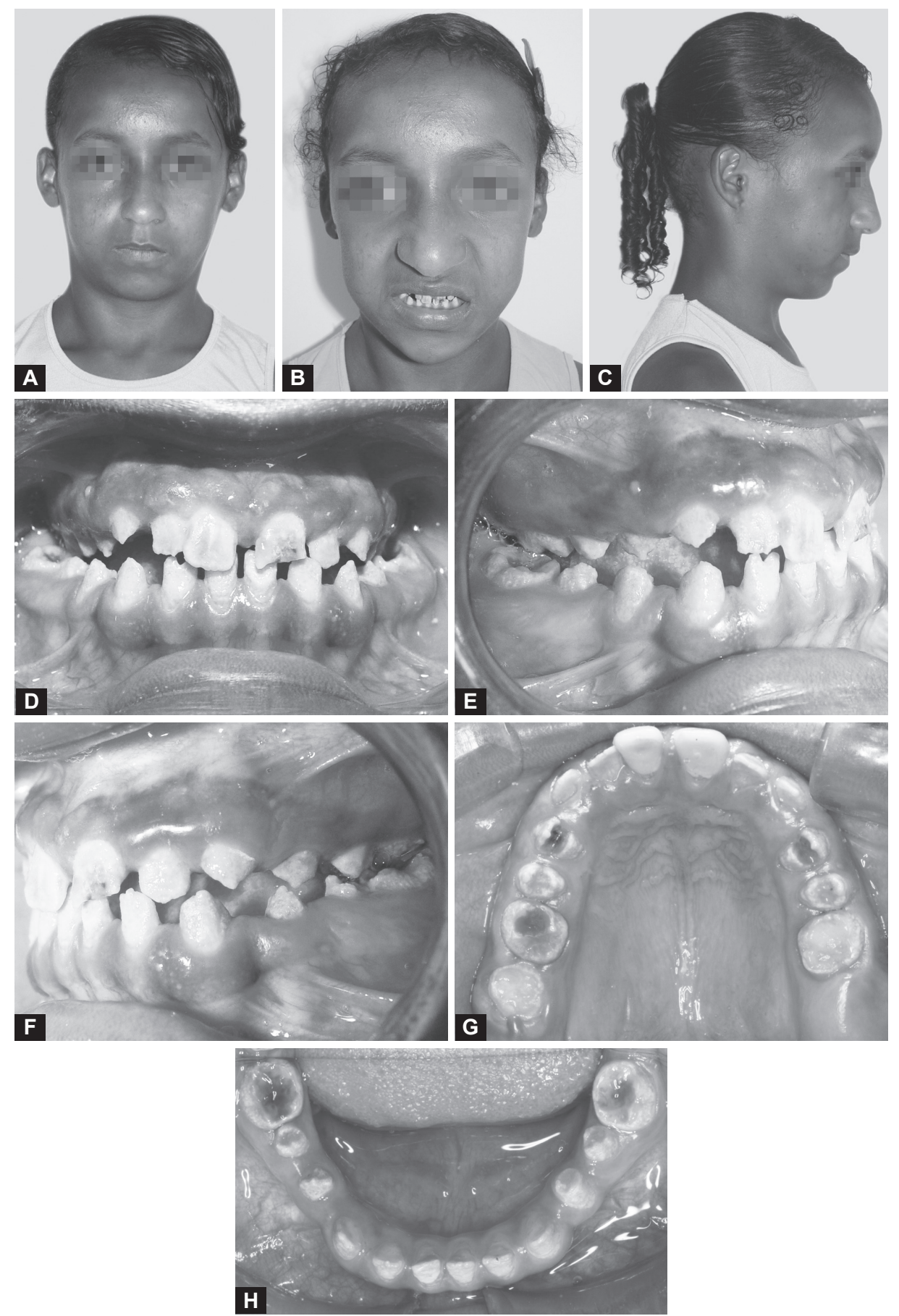

Figs $1 \mathrm{~A}$ to $\mathrm{H}$ : Pretreatment photographs 


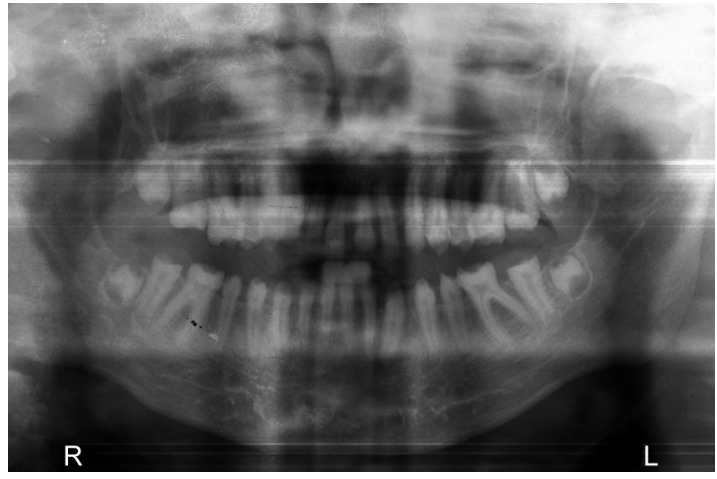

Fig. 2: Pretreatment radiograph
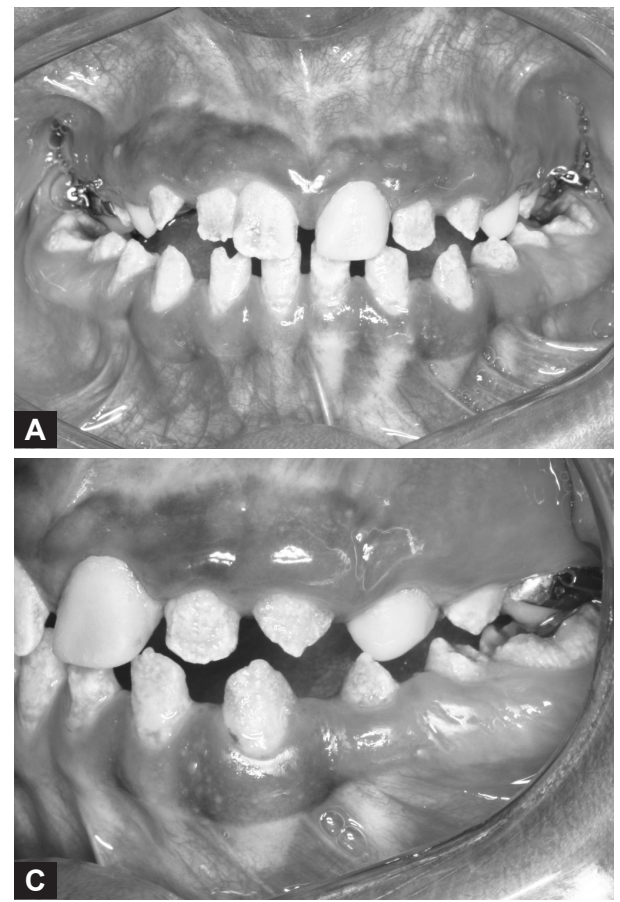

Figs 4A to D: During the treatment with Hass appliance

was performed. In the remaining teeth was planning to restore the end of treatment since the patient had no sensitivity only esthetic. These teeth would receive the orthodontic rings, components of the Hass type (dentalmucus-supported) maxillary expansion appliance. These teeth were reconstructed with resin composite by means of the direct technique. This initial stage was well accepted by the patient, and was of great importance in her psychological re-structuring for continuity of the treatment. Activation of the Hass type appliance was performed for a period of 27 days with $2 / 4$ turns per day, totaling $13 \mathrm{~mm}$ of opening of the suture (Figs 4A to D). After this the appliance was stabilized for 6 months. In this period of stabilization, the stage of fabricating the restorations of the other teeth began.

As an irrefutable detail of the restorative technique using resin composite is the use of absolute isolation, in each section, the teeth to be restored were isolated and had ties
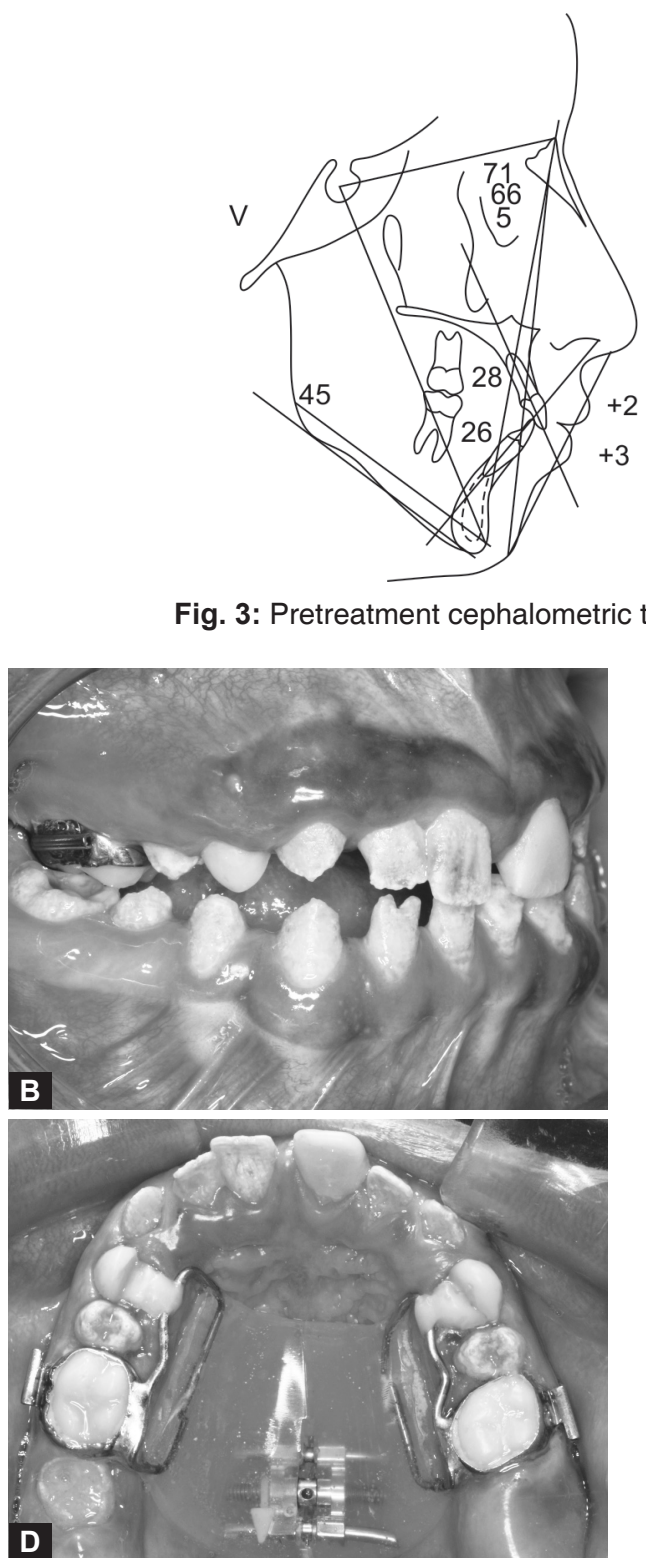

Fig. 3: Pretreatment cephalometric tracing

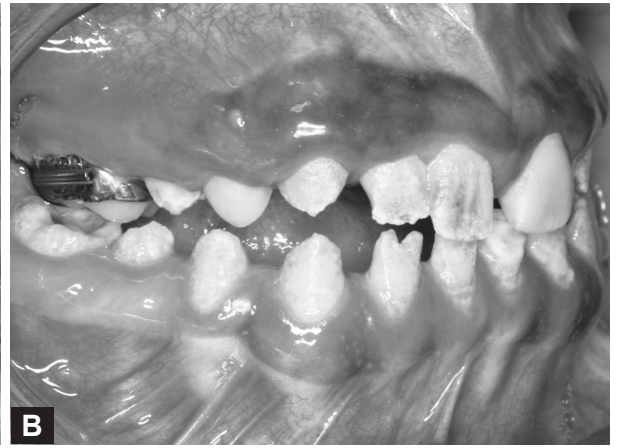

made of dental floss on the gingival limits. After this, the use of a one-bottle adhesive system single Bond (3M-ESPE, St Paul, USA) began, according to the manufacturer's instructions. After dentinal hybridization, it was the correct time to start the direct restorations with resin composite (3M-ESPE, St Paul, USA), using the diagnostic waxing as a guide for correct shaping. Finishing and polishing were performed seven days after each section had been restored, using ultrafine diamond tips (KG Sorensen, São Paulo, Brazil) and sequential finishing disks (Soflex Pop On, 3M-ESPE, St Paul, USA).

Once the teeth had been restored and the period of 6 months after stabilization of the Hass type appliance had elapsed, the appliance was removed, and then the posterior teeth that had supported the appliance were restored to harmonize with the other teeth. Cephalometrically the end of treatment the patient improved facial and maxillo- 

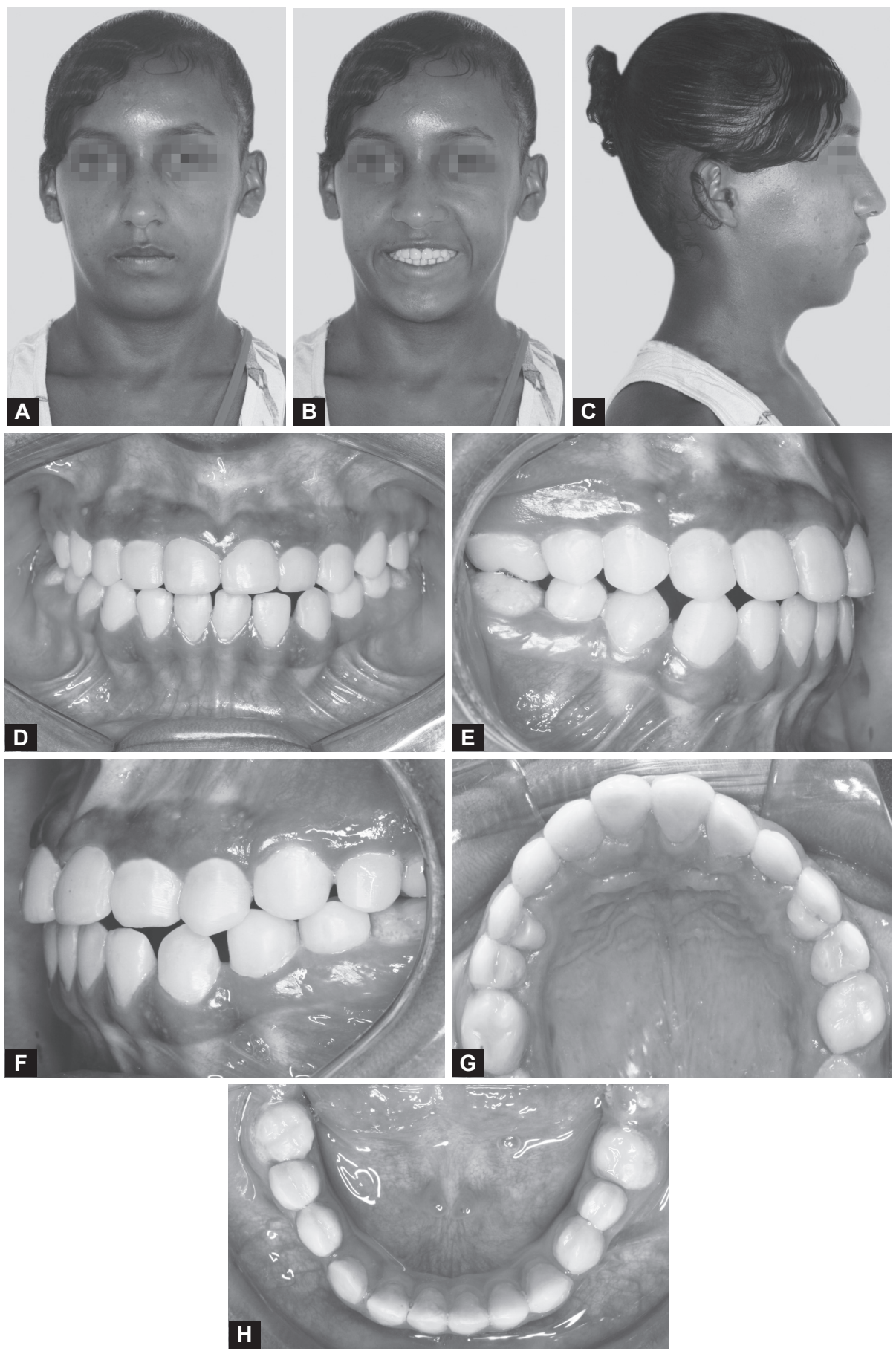

Figs $5 \mathrm{~A}$ to $\mathrm{H}$ : Post-treatment photographs

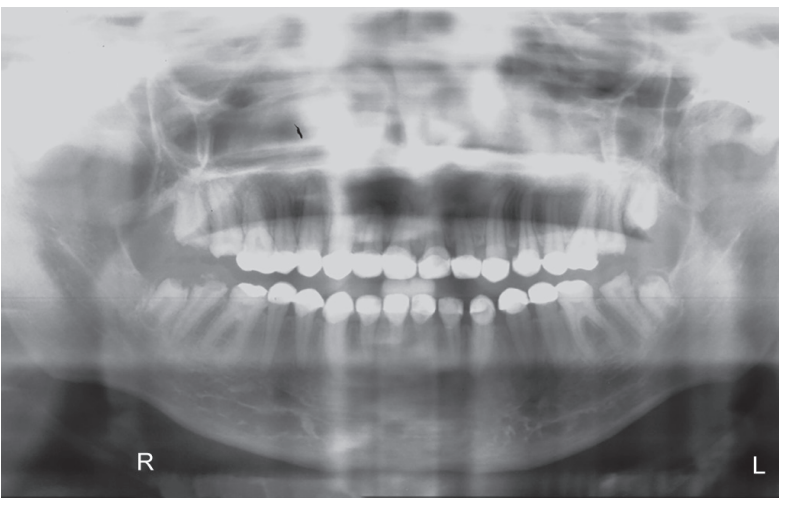

Fig. 6: Post-treatment radiograph mandibular relationship $\left(\mathrm{SNA}=73^{\circ}, \mathrm{SNB}=70^{\circ} \mathrm{eANB}=1^{\circ}\right)$ At the present time, the patient is being followed up until the adult stage, when new procedures should be performed Figs 5 to 8 ).

\section{DISCUSSION}

Amelogenesis imperfecta, initially described in 1890, is distinguished as a set of disorders of a hereditary nature, which presents developmental abnormalities in the quantity and/or quality of tooth enamel, and absence of manifestations of a general or systemic type. ${ }^{9}$ The enamel present in 


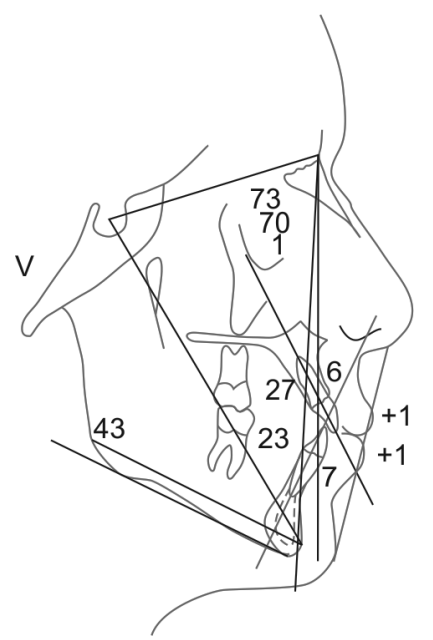

Fig. 7: Post-treatment cephalometric tracing

the tooth is a tissue of ectodermic origin. The enamel organ, known as amelogenesis, is responsible for the composition of tooth enamel. Fundamentally, amelogenesis comprises two stages: in the secretion stage, the cells of the internal epithelium of enamel are characterized as ameloblasts after dentin deposition by the odontoblasts, and in the maturation stage, in which greater mineral addition occurs together with the removal of organic matter and water. ${ }^{10,11}$

The protein amelogenin participates in the constitution of enamel, acting as an insoluble matrix, in which hydroxyapatite crystal deposition occurs during the enamel mineralization stage. Amelogenesis imperfecta is a heterogeneous and autosomal disease; it may be dominant, recessive or linked to Chromosome $\mathrm{X}$, and in the latter case, it is directly linked to the amelogenin gene..$^{10,11}$

The presence of factors, such as nutritional deficiencies (lack of vitamins A, C and D), exanthematous diseases (measles, varicella, scarlet fever), congenital syphilis, hypocalcemia, birth trauma, prematurity, fetal erythroblastosis, infection or local traumatism, ingestion of chemical substances (particularly fluoride) and idiopathic causes may lead to causing damage to enamel formation. ${ }^{4-6}$ In anamnesis, nothing was related by the mother, however, as the patient was from a decidedly underprivileged economic level, it is suggested that this problem resulted from extreme nutritional deficiencies the patient suffered in childhood.

The clinical problems that predominate in amelogenezis imperfecta are the dephasing of dental tissue, compromised esthetics and dentin sensitivity. ${ }^{12,13}$ The pulp and dentinal tissue do not undergo alterations and the teeth have considerable resistance to caries disease, as may be proved in the present case. Enamel roughness favors plaque retention and calculus formation, leading to the inflamed gingiva being a common clinical factor among patients with amelogenesis imperfecta; $, 5,13$ and since these characteristics were remarkable in the patient in this report, they denoted a precarious

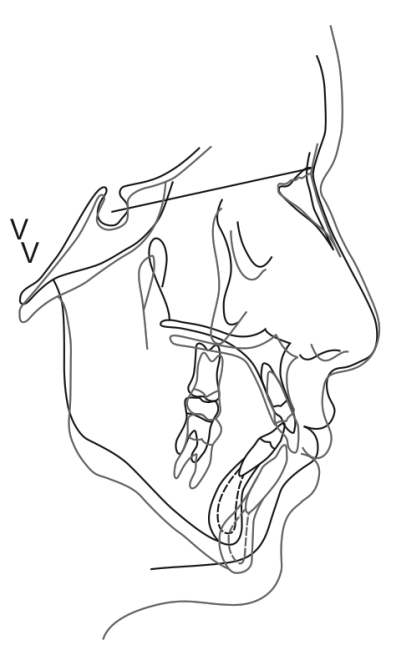

Fig. 8: Superimposed cephalometric tracings

psychological condition due to the influence of the problems resulting from this disturbance.

The variation ranges from an aspect of deficient enamel through to episodes of defects in the mineral and protein substance, and may be identified based on the clinical, radiographic and histological characteristics. ${ }^{12}$ The hypoplasic type involves a deficiency in quantity. Thus, there are no alterations as regards calcification, but the enamel thickness is reduced in some areas. In the hypocalcified type, the enamel is constituted of a matrix without alteration, but is completely calcified. The hypomature type is determined by failures in the final deposition of crystals and development of enamel. ${ }^{12}$ This tissue has a stained and slightly softened aspect in comparison with unaltered enamel, however, it is of normal thickness. ${ }^{12}$ According to the classification into which this clinical case fits, the teeth may be extremely sensitive to thermal and chemical stimuli. ${ }^{5}$ In children, the frequency of episodes occurs in 60 to $70 \%$ of cases in the hypoplasic type, 20 to $40 \%$ in the hypocalcified and $7 \%$ in the hypomatured type. ${ }^{13}$ Therefore, after analysis of all the clinical and radiographic characteristics present in this clinical case, amelogenesis imperfecta of the hypoplasic type with complete absence of tooth enamel, could be confirmed.

Amelogenesis imperfecta has been associated with abnormalities of tooth eruption, congenital absence of teeth, anterior open occlusal relationship, pulp calcification, root and coronal resorption, hypercementosis, root malformations and taurodontism. ${ }^{13}$ The patient, whose case is here related, presented openbite and posterior crossbite in addition to alteration in enamel of all the teeth. To resolve this clinical situation, facial orthopedics with palatal expansion was used to solve the orthopedic problem, with correction of the posterior crossbite associated with a restorative treatment to resolve the esthetics of the teeth, with closure of the posterior open bite. 
Palatal expansion is a resource of great value in orthodontic practice; by means of rupturing the median palatal suture and disorganization of the other craniofacial complex sutures, it enables uncrossing of the posterior bite and increasing the perimeter of the maxillary arch, among other alterations. ${ }^{14}$ After orthopedic treatment using the device type Haas marked improvement in maxillo-mandibular positioning. Despite gains esthetic and functional at the end of treatment, the patient still need to go through a treatment with braces to correct the root position and improve the forms of the upper and lower arches.

In such cases, the expansion of the maxillary arch promotes muscle pull (orofacial and masticatory) in the most appropriate direction, maximizing the growth of the orofacial muscles and providing favorable effect on growth of the jaws, tooth alignment and esthetics dentolabial. Expanding the upper arch, you can create an excellent condition for which the jaw can express their full growth, aiding in the correction of class II. ${ }^{15}$

The successful treatment of class II malocclusions in children is related to the use of normal growth and potential to interfere with this growth through appropriate mechanical. ${ }^{16}$

The treatment of cases of amelogenesis imperfecta may be part of a complex restorative treatment plan and may depend on the severity of the condition. Interdisciplinary treatment options combine orthodontic therapy, orthognathic surgery, periodontal surgery and dental prosthesis. ${ }^{17}$

According to $\mathrm{Hu}$, the enormous progress of esthetic Dentistry, especially as regards dental adhesives, today allow function and esthetics to be rehabilitated up to the point of clinically admissible conditions. ${ }^{4}$

Although, there are innumerable treatments ranging from total crowns (cast, metal-ceramic, total metal), through to extraction of all the teeth in order to fabricate complete dentures; the use of adhesives and resin composites for the treatment of amelogenesis imperfect, allied to expansion of the maxilla with the Hass type orthodontic appliance, appeared to us to be the most coherent therapy, and the one indicated for this case. ${ }^{13}$

It is worth emphasizing that according to Seow, 1993, the fabrication of total crowns made of porcelain as permanent esthetic restorations, are probably the treatment of choice for the oral rehabilitation of patients with AI. However, their use in youngsters, according to this author, is contraindicated due to the presence of pulp chambers with large dimensions. ${ }^{18}$

\section{CONCLUSION}

Interdisciplinary treatment in cases of amelogenesis imperfecta is imperative for correct diagnosis and adequate treatment. Facial orthopedics associated with dentistry, in the case here described, provided improvements from the patient's functional, esthetic and occlusal point of view.

\section{REFERENCES}

1. Fagrell T. Molar incisor hypomineralization. Morphological and chemical aspects, onset and possible etiological factors. Swed Dent J Suppl 2011;5(216):11-83.

2. Li L, Li J. Investigation of molar-incisor hypomineralization among children from 6 to 11 years in Lucheng district, Wenzhou city. Shanghai Kou Qiang Yi Xue 2012;21(5):576-579.

3. Kellerhoff NM, Lussi A. 'Molar-incisor hypomineralization'. Schweiz Monatsschr Zahnmed 2004;114(3):243-253.

4. Hu JC, Chan HC, Simmer SG, Seymen F, Richardson AS, Hu Y, et al. Amelogenesis imperfecta in two families with defined AMELX deletions in ARHGAP6. PLoS One 2012;7(12):520-552.

5. Ardu SS, Duc O, Krejci I, Perroud R. Amelogenesis imperfecta: a conservative and progressive adhesive treatment concept. Oper Dent 2012;38(3):235-241.

6. Yaprak E, Subasi MG, Avunduk M, Aykent F. Amelogenesis imperfecta and generalized gingival overgrowth resembling hereditary gingival fibromatosis in siblings: a case report. Case Rep Dent 2012;2012:423-428.

7. Finkelstein T, Shapira Y, Shpack N. Nonsurgical treatment of severe open bite associated with amelogenesis imperfecta. J Clin Orthod 2012;46(7):427-433; quiz 438.

8. Turkun LS. Conservative restoration with resin composites of a case of amelogenesis imperfecta. Int Dent J 2005;55(1):38-41.

9. Wright JT, Aldred MJ, Crawford PJ, Kirkham J, Robinson C. Enamel ultrastructure and protein content in X-linked ameloge-nesis imperfecta. Oral Surg Oral Med Oral Pathol 1993;76(2):192-199.

10. Parry DA, Poulter JA, Logan CV, Brookes SJ, Jafri H, Ferguson $\mathrm{CH}$, et al. Identification of mutations in SLC24A4, encoding a potassium-dependent sodium/calcium exchanger, as a cause of amelogenesis imperfecta. Am J Hum Genet 2013;92(2):307-312.

11. Simmer S, Estrella N, Milkovich R, Hu J. Autosomal dominant amelogenesis imperfecta associated with ENAM frame shift mutation p.Asn36Ilefs56. Clin Genet 2013;83(2):195-197.

12. Tahmassebi JF, Day PF, Toumba KJ, Andreadis GA. Paediatric dentistry in the new millennium: 6. Dental anomalies in children. Dent Update 2003;30(10):534-540.

13. Toksavul S, Ulusoy M, Turkun M, Kumbuloglu O. Amelogenesis imperfecta: the multidisciplinary approach. A case report. Quintessence Int 2004;35(1):11-14.

14. Witkop CJ Jr. Amelogenesis imperfecta, dentinogenesis imperfecta and dentin dysplasia revisited: problems in classification. J Oral Pathol 1988;17(9-10):547-553.

15. Lima Filho RM, de Oliveira Ruellas AC. Mandibular behavior with slow and rapid maxillary expansion in skeletal class II patients: a long-term study. Angle Orthod 2007;77(4):625-631.

16. Evans CA. Anteroposterior skeletal change: Growth modification. Semin Orthod 2000;6(3):21-32.

17. Ozturk N, Sari Z, Ozturk B. An interdisciplinary approach for restoring function and esthetics in a patient with amelogenesis imperfecta and malocclusion: a clinical report. J Prosthet Dent 2004;92(2):112-115.

18. Seedorf H, Springer IN, Grundner-Culemann E, Albers HK, Reis A, Fuchs H, et al. Amelogenesis imperfecta in a new animal model-a mutation in chromosome 5 (human 4q21). J Dent Res 2004;83(8):608-612. 